\title{
Atorvastatin inhibits endoplasmic reticulum stress through AMPK signaling pathway in atherosclerosis in mice
}

\author{
WANGQIONG XIONG $^{1 *}$, MINZHONG FEI ${ }^{2 *}$, CHUNTAO WU $^{2}$, WEI WANG ${ }^{2}$, \\ RONG LUO $^{2}$, LIPING SHEN ${ }^{1}$ and ZHENG ZHANG ${ }^{2}$ \\ ${ }^{1}$ ECG Room and ${ }^{2}$ Cardiovascular Department, Qingpu Branch of Zhongshan Hospital Affiliated to Fudan University, \\ Shanghai 201700, P.R. China
}

Received October 4, 2019; Accepted December 4, 2019

DOI: $10.3892 / \mathrm{etm} .2019 .8379$

\begin{abstract}
Effect of atorvastatin inhibition of endoplasmic reticulum stress and amelioration of atherosclerosis through AMPK pathway were studied. Eight-week-old male apolipoprotein E-deficient (ApoE $\left.{ }^{-/}\right)$mice were fed with high-fat diet for 2 weeks and randomly divided into two groups: Atorvastatin treatment group was given atorvastatin ( $5 \mathrm{mg} / \mathrm{kg}$ /day) injection for a total of 6 weeks; control group was given the same dose of PBS through intraperitoneal injection for a total of 6 weeks. H\&E staining was used to detect plaque size; immunohistochemical staining was used to detect T cells, macrophages and phospho-protein kinase-like ER kinase (phospho-PERK) in localized plaques. Proteins were extracted from mouse thoracic and abdominal aortic tissues. Western blot analysis was used to detect the protein expression levels of endoplasmic reticulum stress-related molecules phospho-eukaryotic initiation factor- $2 \alpha$ (p-eIF $2 \alpha$ ), eukaryotic initiation factor (eIF2a), and sliced x-box binding protein 1 (sXBP-1). Cultured human umbilical vein endothelial cells (HUVECs), induced endoplasmic reticulum stress with human oxidized low density lipoprotein (ox-LDL), were treated with atorvastatin, AMPK agonist 5-amino-4-imidazolecarboxamide riboside-I- $\beta$-D-ribofuranoside (AICAR) and AMPK-DN that expressed a dominant-negative mutant of AMPK. Western blot analysis was used to test the expression levels of endoplasmic reticulum stress-related molecules p-elF2a and sXBP-1. The area of aortic plaques in atorvastatin group was obviously decreased, and the infiltrations of $\mathrm{CD}^{+} \mathrm{T}$ cells and macrophages in the localized plaques were reduced. The endoplasmic reticulum stress-related proteins sXBP-1 and p-eIF2a were significantly reduced. The results of
\end{abstract}

Correspondence to: Dr Zheng Zhang, Cardiovascular Department, Qingpu Branch of Zhongshan Hospital Affiliated to Fudan University, 1158 East Park Road, Qingpu, Shanghai 201700, P.R. China

E-mail: zhangzheng1402@sina.cn

*Contributed equally

Key words: atherosclerosis, atorvastatin, endoplasmic reticulum stress, AMPK immunohistochemistry also showed a significant decrease in the level of phospho-PERK (p-PERK) in atorvastatin group. The results in ox-LDL-induced HUVECs showed that atorvastatin inhibited ox-LDL-induced endoplasmic reticulum stress, and the AMPK agonist AICAR also had the same effect, which was offset by DN-AMPK treatment. Atorvastatin inhibits ER stress both in vitro and in vivo and this protective effect is mediated by AMPK activation.

\section{Introduction}

Atherosclerosis (AS) and the resulting coronary heart disease (CHD) are threatening human health both in developed and developing countries. In China, with the improvement of people's living standards and changes in dietary habits, the incidence rates of AS and its related diseases also show an increasing trend year by year, which not only seriously endangers people's health, but also lay as huge economic burden on the society. Therefore, it is extremely urgent to fully understand the pathogenesis of AS and CHD. It is well known that the occurrence of AS is not simply due to dyslipidemia, but also closely related to chronic inflammatory diseases. The inflammatory responses occur throughout AS, and is one of the main causes of AS, exerting effects on the progression of AS mainly through affecting lipid metabolism and biological activity of the vascular wall (1).

The complex interaction among arterial vascular cells, lipoproteins and inflammatory cells is involved in AS. The key factor in the initiation of AS is the abundance of lipoprotein in apolipoprotein B under the endothelium, followed by recruitment and differentiation of inflammatory monocytes into macrophages (2). Macrophages take in lipoproteins and turn into lipid-loaded 'foam cells', which gradually exacerbate the inflammatory state along with other immune cells and intimal smooth muscle cells. The dead and dying macrophages, combined with insufficient scavenging ability of dead cells, lead to development of the so-called lipid-rich necrotic core, which is a complex process closely related to the rupture of atherosclerotic plaques, thereby causing acute myocardial infarction and sudden death (3). The lipid-rich necrotic core is associated with plaque instability, probably because it is the aggregation place of matrix metalloproteinase, inflammatory mediators and pro-thrombosis molecules. Therefore, 
apoptosis of macrophages may be a key factor for facilitating the formation of lipid-rich necrotic core and promoting the transformation of benign pathological changes into unstable lesion phenotype. In advanced AS, an important cause of macrophage apoptosis is the cell death caused by chronic endoplasmic reticulum stress (ERS) pathway (4). The effect of ERS on cells in AS is poorly understood, but currently there is evidence that ERS can regulate the survival of smooth muscle cells and endothelial cells $(5,6)$.

Recent studies have confirmed that adenosine monophosphate-activated protein kinase (AMPK) is a potential target for resisting AS. AMPK is a kind of important protein kinase, which, in the case of cascade activation, will enhance the protective effect of cells under stress state. The main target cells in cardiovascular diseases are cardiovascular endothelial cells and smooth muscle cells, and they are regulated by AMPK directly and indirectly. Most of the evidence comes from the research results on the close association between dysregulation of AMPK pathway and vascular disease. Therefore, AMPK is a key target in the prevention and treatment of AS (7). Studies have demonstrated that the activity of AMPK significantly increases when many cardiovascular organs have no adequate blood flow in the early stage under such acute and chronic stimuli as load increase, but the activation of AMPK will be inhibited by excessive injury, especially under the action of reactive oxygen species (ROS) (8). Therefore, activating the AMPK pathway has become a potential target for drug therapy of AS.

Atorvastatin is a clinically common selective 3-hydroxy-3 - methylglutaryl coenzyme A (HMG-CoA) reductase inhibitor. In addition to its lipid-regulating effect in resisting $\mathrm{AS}$, atorvastatin also slows down the occurrence and development of AS through non-lipid lowering effects, such as anti-inflammation, anti-oxidation and immunoregulation $(9,10)$. It is known that atorvastatin can promote the phosphorylation of AMPK (10), while the AMPK pathway can reduce the adhesion of inflammatory cells to the vascular endothelium and the proliferation of inflammatory cells caused by lipid oxidation, thereby stimulating the expression of related genes in antioxidant defense system in cells and the production of endothelial nitric oxide synthase (11), and exerting the corresponding anti-AS effect.

To better determine the anti-AS mechanism of atorvastatin, it is hypothesized that atorvastatin can reduce the ERS in endothelial cells in apolipoprotein $\mathrm{E}(\mathrm{ApoE})^{-/}$mice, and such a protective effect of atorvastatin is realized through activating AMPK.

\section{Materials and methods}

Animals and reagents. A total of 20, 8-week-old male Specific Pathogen Free (SPF) ApoE ${ }^{-/}$mice, weighing 18-22 g, were from Beijing Vital River Laboratory Animal Technology Co., Ltd. High-fat diet (containing $0.25 \%$ cholesterol and $21 \%$ lard) was from Beijing Keao Xieli Feed Co., Ltd. Rabbit anti-phospho-PERK antibody, and spliced XBP-1 antibody, phosphorylated eIF2a antibody were from Cell Signaling Technology, oxidized low-density lipoprotein (ox-LDL) was from Yiyuan Biotechnologies, and H\&E staining kit was from Beyotime Biotechnology.
Modeling and grouping. This study was approved by the Animal Ethics Committee of Fudan University Animal Center (Qingpu, China). A total of $20 \mathrm{ApoE}^{-/}$mice were fed with high-fat diet added with $0.15 \%$ cholesterol and $21 \%$ lard for 2 weeks to establish the mouse model of hyperlipidemia. After successful modeling, the mice were randomly divided into control group and atorvastatin group. The mice in atorvastatin group were intraperitoneally injected with atorvastatin solution $(5 \mathrm{mg} / \mathrm{kg}$ ) every day, while those in control group were intraperitoneally injected with the same dose of phosphate-buffered saline (PBS). They were fed under the temperature of $21 \pm 2^{\circ} \mathrm{C}$, relative humidity of $50 \pm 15 \%$ and 12/12 h light/dark cycle. After 6 weeks, the mice were sacrificed for further data analysis.

Hematoxylin and eosin $(H \& E)$ staining. After the mice were sacrificed, the blood vessels were first perfused with PBS and then $4 \%$ paraformaldehyde fixative. The blood vessels in the brachial artery and aortic root were separated, fixed in $4 \%$ paraformaldehyde fixative for $12 \mathrm{~h}$ and embedded in OCT. Then the specimens were serially sliced into 20 sections at an interval of $50 \mu \mathrm{m}$, washed with distilled water for $5 \mathrm{~min}$, stained with hematoxylin for $5 \mathrm{~min}$, and washed with water for $3 \mathrm{~min}$, followed by differentiation with $0.5 \%$ hydrochloric acid alcohol for $20 \mathrm{sec}$, washing with tap water for $2 \mathrm{~min}$, eosin staining for $3 \mathrm{~min}$, and washing with tap water for $1 \mathrm{~min}$ again to remove the excess dye. After dehydration with gradient alcohol, $80 \%$ ethanol for $5 \mathrm{sec}, 95 \%$ ethanol I for $2 \mathrm{~min}$ and 95\% ethanol II for $2 \mathrm{~min}$, absolute ethanol I for $3 \mathrm{~min}$ and absolute ethanol II for $3 \mathrm{~min}$, and transparent reagent I for $5 \mathrm{~min}$ and transparent reagent II for $5 \mathrm{~min}$, the sections were sealed with neutral balsam, and the transparent reagent around the tissues was absorbed using absorbent paper. Then a small drop of balsam was added dropwise at the center of tissues, and carefully covered with a clean cover glass using tweezers. Finally, the morphological structure of aortic vessels and plaques was observed under a microscope.

Immunohistochemistry. The sections were taken from the refrigerator $\left(-80^{\circ} \mathrm{C}\right)$, placed at room temperature for $20 \mathrm{~min}$, soaked in distilled water for $20 \mathrm{~min}$, washed with PBS, immersed in $3 \% \mathrm{H}_{2} \mathrm{O}_{2}$ for $10 \mathrm{~min}$, washed with PBS for 5 min, 3 times, sealed with $5 \%$ goat serum and incubated at room temperature for $20 \mathrm{~min}$. After the serum was removed, the sections were added dropwise with primary antibodies (1:50) for incubation at $4^{\circ} \mathrm{C}$ overnight, washed with PBS for 5 min, 3 times, incubated with horseradish peroxidase-labeled secondary antibodies at $37^{\circ} \mathrm{C}$ for $40 \mathrm{~min}$, and washed again with PBS. After color development using diaminobenzidine (DAB) in the dark, the sections were washed with running water, stained with Harris hematoxylin dye for $2 \mathrm{~min}$, washed again with running water for $30 \mathrm{sec}$, and differentiated with $1 \%$ hydrochloric acid alcohol for 5-10 sec, followed by dehydration with ethanol at different concentrations, and transparentization. Then the sections were added dropwise with neutral balsam and covered with the cover glass. The antigen-positive cells were stained dark brown.

Western blotting. The total proteins required for the assay were extracted from the tissues as follows: Approximately $30 \mathrm{mg}$ of 
tissues were taken, ground with liquid nitrogen using a clean mortar, and lysed with cell lysis buffer $(150 \mu 1 / 30 \mathrm{mg})$ for $30 \mathrm{~min}$, followed by centrifugation at $12,000 \mathrm{x} \mathrm{g}$ for $5 \mathrm{~min}$ at $4^{\circ} \mathrm{C}$. The supernatant was taken and stored at $-80^{\circ} \mathrm{C}$. The protein concentration in the sample was detected using bicinchoninic acid (BCA) colorimetry (Pierce; Thermo Fisher Scientific, Inc.). Protein diluent $(25 \mu \mathrm{l})$ and $25 \mu \mathrm{l}$ of standards were added into each well, $200 \mu \mathrm{l}$ of developing solution $(\mathrm{A}: \mathrm{B}=50: 1)$ was also added, and the mixture was incubated in an incubator at $37^{\circ} \mathrm{C}$ for $30 \mathrm{~min}$. The optical density value was measured using a microplate reader, and the concentration and loading volume of the protein sample were calculated. The samples were taken out from the refrigerator $\left(-80^{\circ} \mathrm{C}\right)$ and placed on ice. Electrophoresis solution $(500 \mathrm{ml})$ already prepared was poured into the electrophoresis tank, and the electrophoresis gel was also placed into the electrophoresis tank. The protein was loaded and subjected to electrophoresis under constant pressure of $100 \mathrm{~V}$ for $30 \mathrm{~min}$. After the sample ran through the spacer gel, electrophoresis was performed again under constant pressure of $120 \mathrm{~V}$ for $\sim 1.5 \mathrm{~h}$, after which the gel plate was taken out. The polyvinylidene fluoride (PVDF) membranes (Millipore) was first immersed in methanol for $10 \mathrm{sec}$ and in distilled water for $2 \mathrm{~min}$, and then placed into the transfer buffer. The transfer holder was opened, and the fiber pad, filter paper, PVDF membranes, electrophoresis gel, filter paper and fiber pad were placed in order from bottom to top. Then the transfer holder was placed in the transfer tank containing transfer buffer for membrane transfer. After the electrode was connected, the protein was transferred onto the membrane under constant pressure of $80 \mathrm{~V}$ for $2 \mathrm{~h}$, sealed with $5 \%$ skim milk for $2 \mathrm{~h}$, and incubated with primary antibodies overnight. After the membrane was washed, the protein was incubated again with secondary antibodies for $1 \mathrm{~h}$, and the color was developed.

Statistical analysis. GraphPad Prism 6.0 software (GraphPad Software, Inc.) was used for plotting, and Statistical Product and Service Solutions (SPSS) 22.0 software (IBM, Corp.) for statistical analysis. Measurement data were expressed as mean \pm standard deviation (mean \pm SD). Differences between two groups were analyzed by using the Student's t-test. Comparison between multiple groups was done using One-way ANOVA test followed by Post Hoc Test (Least Significant Difference). $\mathrm{P}<0.05$ indicates a statistically significant difference.

\section{Results}

Atorvastatin treatment reduces atherosclerotic plaque formation and increases plaque stability in ApoE ${ }^{-}$mice. To detect the effect of atorvastatin on atherosclerotic plaque formation, the aortic root sections were H\&E stained. The results showed that the area of atherosclerotic plaques in the thoracic aorta were significantly reduced in atorvastatin group $(\mathrm{P}<0.05)$ (Fig. 1A), indicating that atorvastatin applied in vivo can reduce the atherosclerotic plaque formation. To explore the effect of atorvastatin on plaque stability, the aortic root sections of $\mathrm{T}$ cells and macrophages were stained. The results revealed that the content of macrophages declined, and the $\mathrm{CD} 3^{+} \mathrm{T}$ cell infiltration was also significantly reduced in atorvastatin group
$(\mathrm{P}<0.05)$ (Fig. 1B), suggesting that the plaque stability in the atorvastatin group was significantly higher than that in the control group.

Atorvastatin alleviates ERS in local vascular walls and plaques in ApoE\% mice. The level of ERS in local vascular walls and plaques was first detected after intraperitoneal injection of atorvastatin. The thoracic aorta was ground and smashed to extract the proteins, and the ERS-related proteins spliced X box-binding protein 1 (sXBP-1) and phospho-eukaryotic initiation factor- $2 \alpha$ (p-eIF $2 \alpha$ ) were selected as detection markers for ERS and subjected to western blotting. It was found that the levels of ERS-related proteins sXBP-1 and p-eIF2 $\alpha$ were obviously reduced in the thoracic aorta in atorvastatin group, while the level of total eIF $2 \alpha$ had no changes (Fig. 2A), demonstrating that atorvastatin can effectively inhibit activation of the above proteins, alleviating the occurrence of ERS. To clarify the local conditions of plaques, immunohistochemistry was used to detect the level of another ERS-related protein phospho-protein kinase-like ER kinase (p-PERK) in local plaques. The results showed that the level of $\mathrm{p}$-PERK in local plaques obviously declined $(\mathrm{P}<0.05)$ (Fig. 2B). The above findings indicate that atorvastatin applied in vivo can relieve ERS in local vascular walls and plaques.

Atorvastatin attenuates ox-LDL-induced ERS in human umbilical vein endothelial cells (HUVECs) through activating $A M P K$. HUVECs were divided into control (NC) group, ox-LDL group (treated with ox-LDL at a concentration of $100 \mu \mathrm{g} / \mathrm{ml}$ for $24 \mathrm{~h}$ ) and atorvastatin group (treated with ox-LDL at a concentration of $100 \mu \mathrm{g} / \mathrm{ml}$ and atorvastatin at a concentration of $10 \mu \mathrm{mol} / 1$ for $24 \mathrm{~h}$ ), and the levels of ERS-related molecules p-elF2a and sXBP-1 were determined using western blotting. It was found that the levels of p-elF2a and sXBP-1 were increased in ox-LDL group, while the elevated protein levels declined in atorvastatin group $(\mathrm{P}<0.05)$ (Fig. 3A). Furthermore, HUVECs were divided into ox-LDL group, atorvastatin group and AMPK agonist 5-aminoimidazole-4-carboxamide-I- $\beta$-D-ribofurano side (AICAR) group (treated with ox-LDL at a concentration of $100 \mu \mathrm{g} / \mathrm{ml}$ and $1 \mathrm{mM}$ of AICAR for $24 \mathrm{~h}$ ). The results manifested that after application of atorvastatin and AMPK agonist AICAR, the level of AMPK was increased, and the protein expression levels of ERS-related molecules were also inhibited in AICAR group, indicating that AMPK agonist and atorvastatin are able to exert the same inhibitory effect on ox-LDL-induced ERS in HUVECs $(\mathrm{P}<0.05)$ (Fig. 3B). To explore the role of AMPK in the inhibition of atorvastatin on ERS, HUVECs were transfected with AMPK-DN (treated with ox-LDL at a concentration of $100 \mu \mathrm{g} / \mathrm{ml}$ and AICAR plasmid for $24 \mathrm{~h}$ ). The results showed that AMPK-DN could effectively suppress the atorvastatin-induced increase of AMPK level, and in AMPK-DN group, the inhibitory effect of atorvastatin on ox-LDL-induced ERS in HUVECs was evidently weakened, and the decreased protein levels of ERS-related molecules p-elF2a and sXBP-1 increased again $(\mathrm{P}<0.05)$ (Fig. 4A and $\mathrm{B})$. It is concluded that atorvastatin inhibits the ox-LDL-induced ERS in HUVECs through AMPK. 

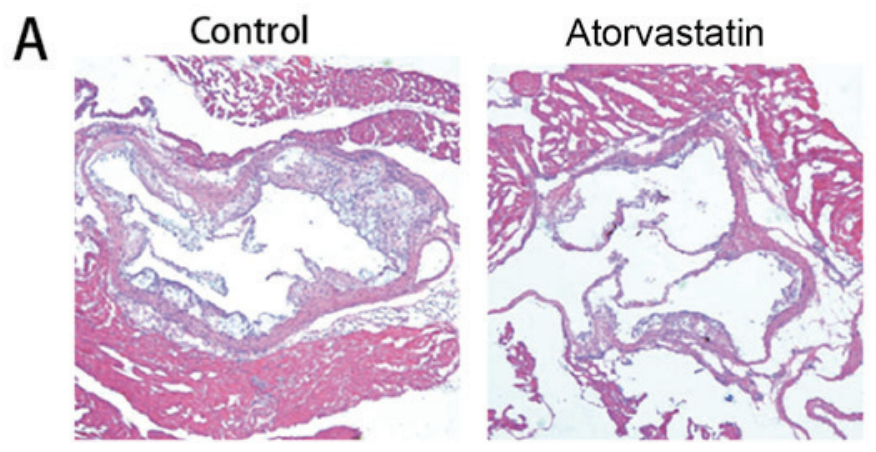

B
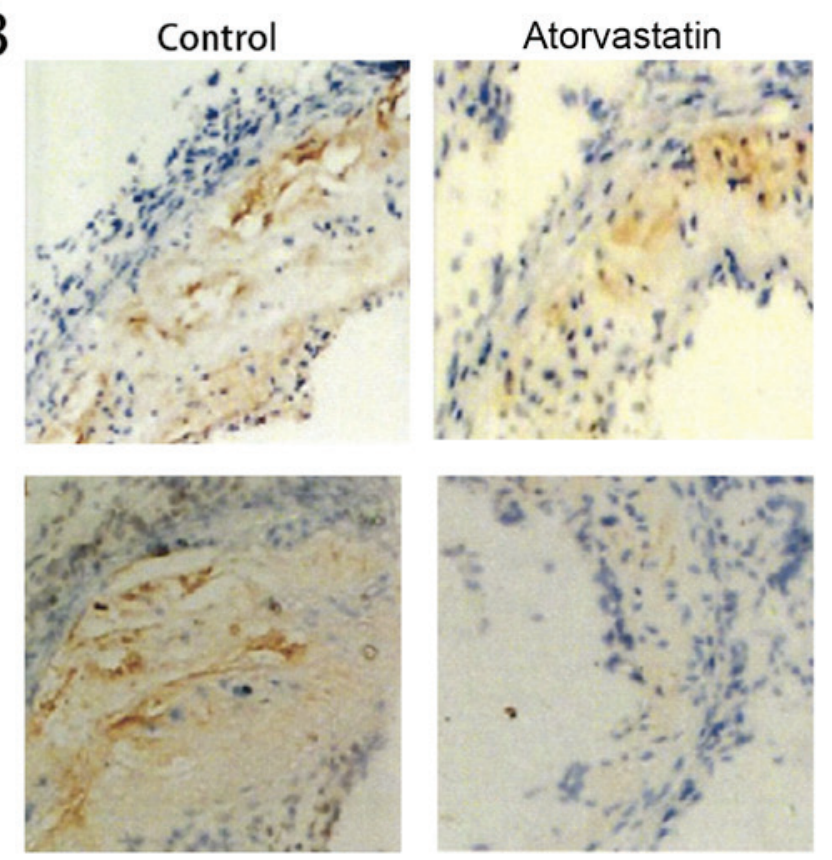
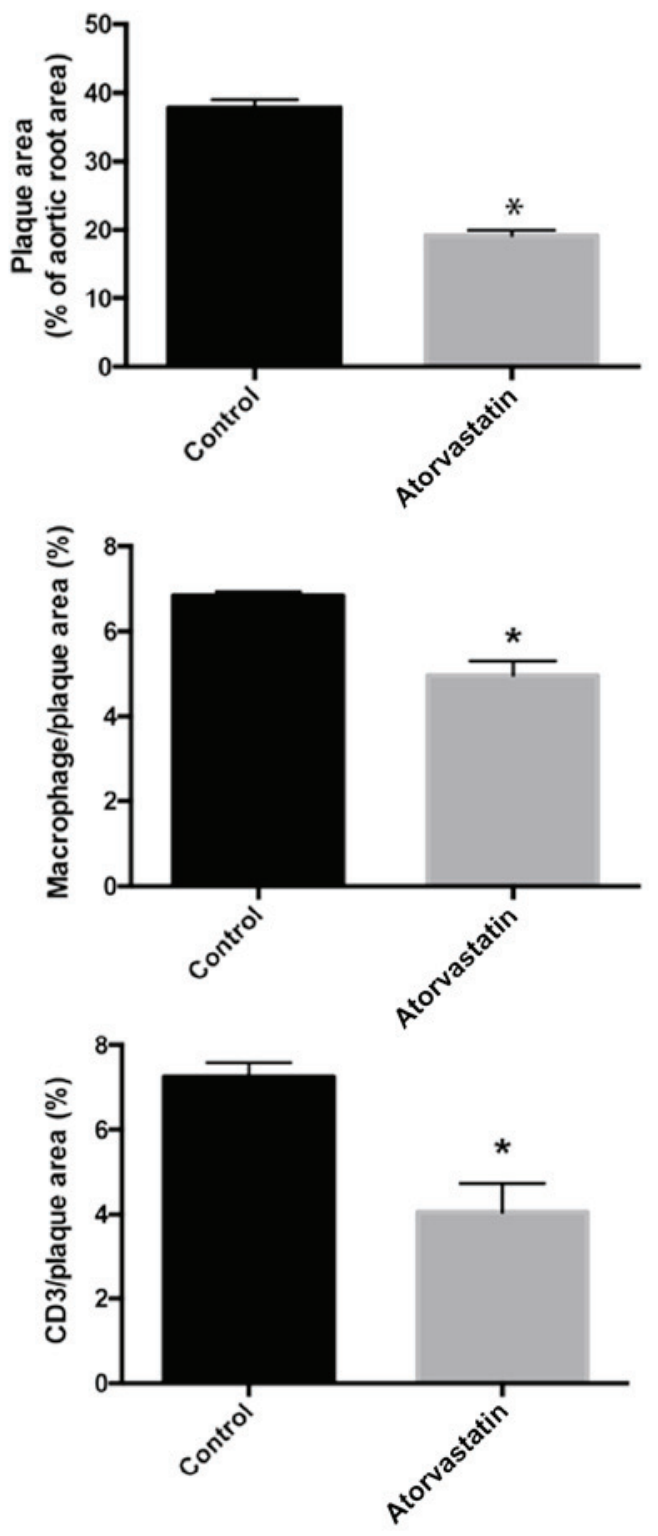

Figure 1. Atorvastatin treatment reduces atherosclerotic plaque formation and increased plaque stability in ApoE ${ }^{-/-}$mice. (A) Serial sections of the aortic roots from $\mathrm{ApoE}^{-/}$mice (control) and atorvastatin-treated $\mathrm{ApoE}^{-/-}$mice (atorvastatin) were stained with H\&E and observed under light microscopy. The size of atherosclerotic lesions was measured and quantified. ${ }^{*} \mathrm{P}<0.05$ atorvastatin vs. control. Scale bar, $200 \mathrm{~mm}$. (B) Serial sections of the aortic roots from ApoE ${ }^{-/}$ mice (control) and atorvastatin-treated $\mathrm{ApoE}^{-/-}$mice (atorvastatin) were stained and expression of CD3 and macrophage in plaque were analyzed and quantified by immunohistochemistry. ${ }^{*} \mathrm{P}<0.05$ atorvastatin vs. control. Scale bar, $100 \mathrm{~mm}$. ApoE ${ }^{-/}$, apolipoprotein E-deficient.

\section{Discussion}

ER is an important organelle and calcium ion reservoir for protein synthesis, folding, modification and transport in eukaryotic cells, and is also closely related to the lipid synthesis and the maintenance of redox balance. ER is very sensitive to a variety of stimuli, such as oxidative stress, imbalance of calcium homeostasis, cholesterol overload, glycosylation changes and other changes in physicochemical environment, which can lead to dysfunction of ER, and cause ERS mainly characterized by accumulation of unfolded and/or misfolded proteins and imbalance of calcium homeostasis. The massive accumulation of unfolded and/or misfolded proteins in the ER lumen will result in the activation of a series of intracellular signal transduction pathways, known as unfolded protein response (UPR). UPR to a certain degree is beneficial for maintaining the ER function and cell survival, but excessive or over long-term stress will induce apoptosis through activating ERS-related signaling pathways (12). Cardio-cerebrovascular diseases with AS as the pathological basis seriously harm human health. In recent years, a large number of basic and clinical studies have confirmed that ERS plays an important role in the occurrence and development of AS, and it is expected to become a new therapeutic target for AS (13). Currently, UPR is the most well-studied ERS signaling pathway, which is sensed and mediated by 3 kinds of ER transmembrane proteins, namely double-stranded RNA-dependent PERK, inositol-requiting enzyme 1 (IRE1) and activating transcription factor 6 (ATF6).

PERK is type I ER transmembrane protein with serine/threonine kinase activity. Under ERS, activated PERK facilitates the phosphorylation of eukaryotic translation 
A

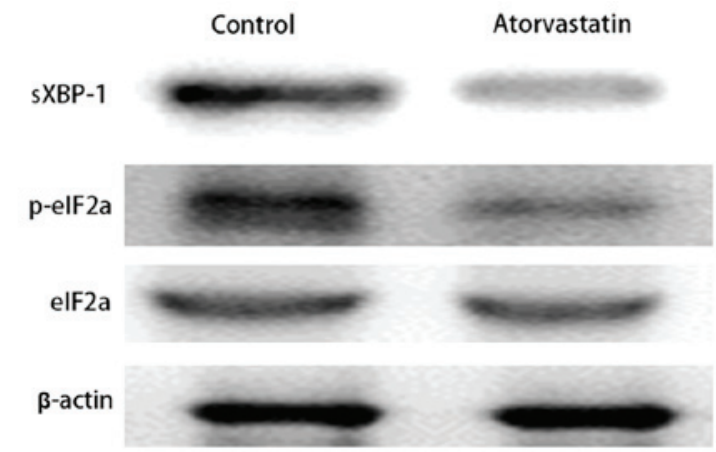

B
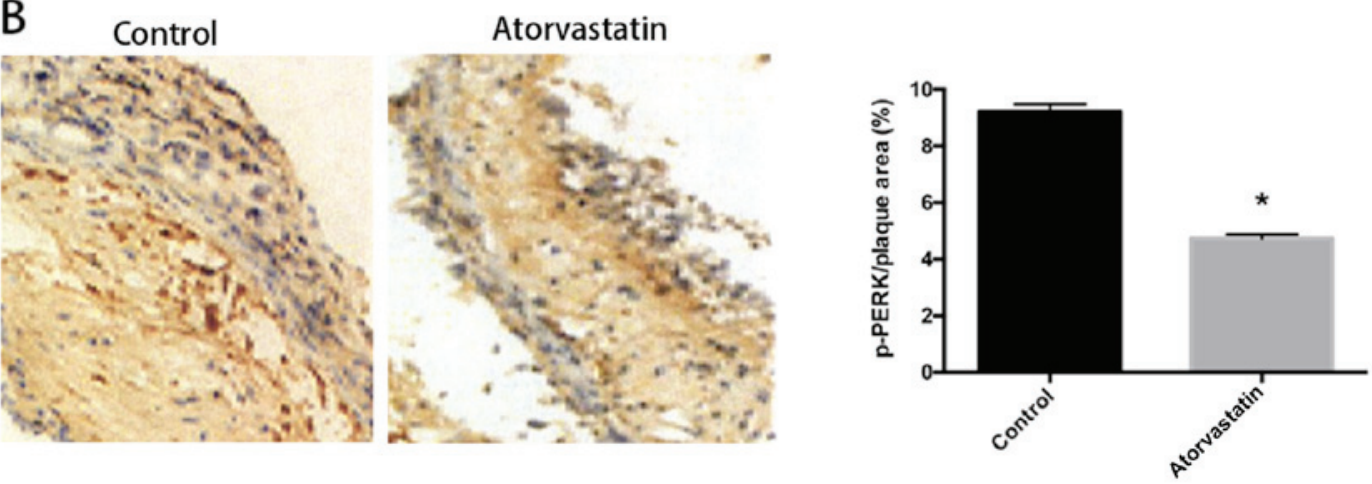

Figure 2. Atorvastatin alleviates ERS in local vascular walls and plaques in $\mathrm{ApoE}^{-/}$mice. (A) Tissue extracts from aortic roots of $\mathrm{ApoE}^{-/-}$mice (control) and atorvastatin-treated $\mathrm{ApoE}^{--}$mice (atorvastatin) were analyzed by western blot analysis. sXBP-1, p-eIF2a and eIF2a protein levels were examined and normalized with $\beta$-actin. (B) The aortic root sections from $\mathrm{ApoE}^{-/}$mice (control) and atorvastatin-treated $\mathrm{ApoE}^{-/}$mice (atorvastatin) were stained by rabbit anti-p-PERK and expression of p-PERK in plaque was analyzed by immunohistochemistry. ${ }^{*} \mathrm{P}<0.05$ atorvastatin vs. control. ApoE ${ }^{-1}$, apolipoprotein E-deficient; sXBP-1, sliced x-box binding protein 1; eIF2a, eukaryotic initiation factor; PERK, protein kinase-like ER kinase.
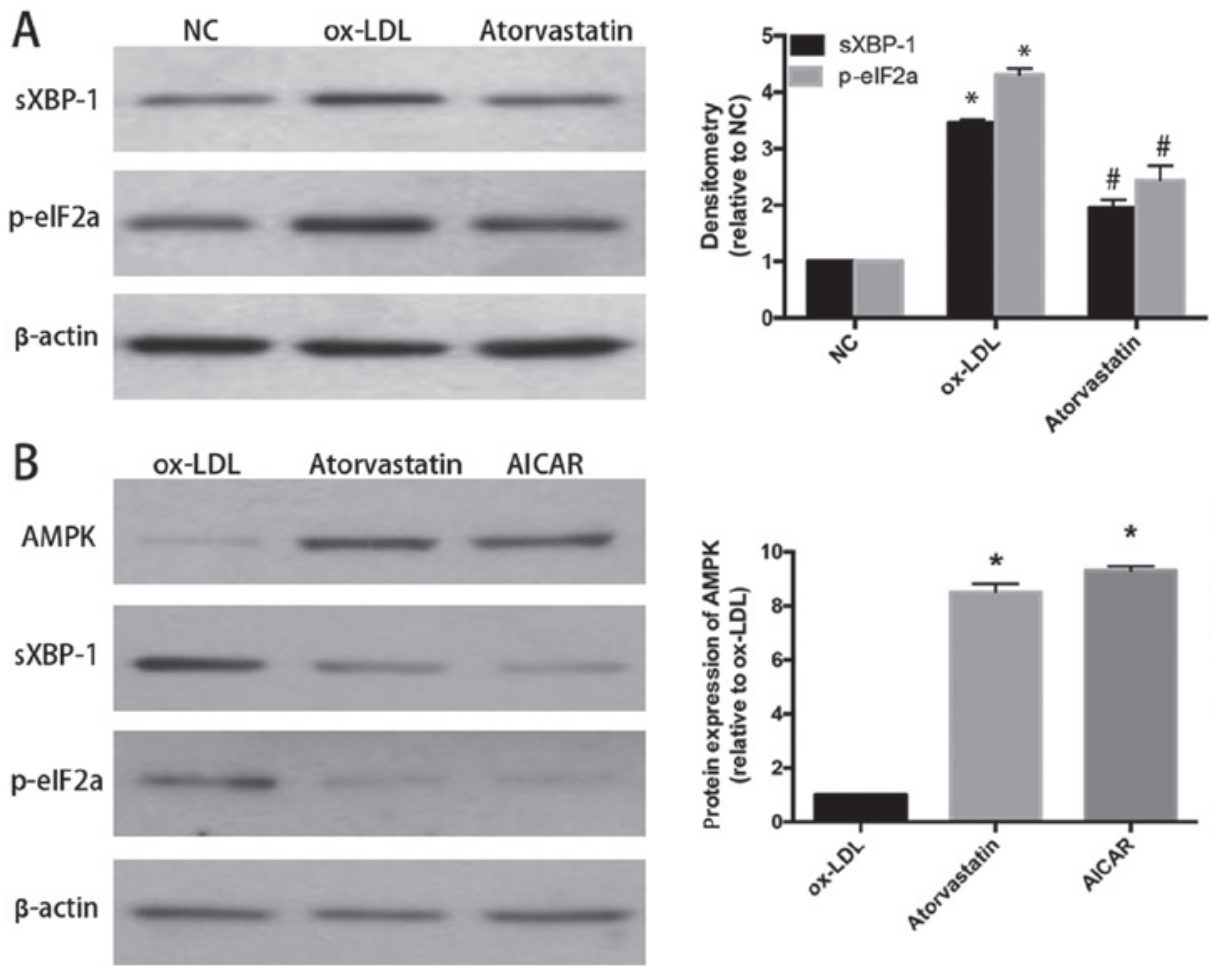

Figure 3. Atorvastatin attenuates oxidized modified low-density lipoprotein (ox-LDL)-induced ERS in human umbilical vein endothelial cells (HUVECs) through activating AMPK. (A) Proteins from HUVECs of control (NC) group, ox-LDL-treated (ox-LDL) group and atorvastatin treated plus ox-LDL-treated (atorvastatin) group were analyzed by western blot analysis. sXBP-1 and p-eIF2a protein levels were examined and quantified. " $\mathrm{P}<0.05$ ox-LDL vs. NC; ${ }^{\text {"}} \mathrm{P}<0.05$ atorvastatin vs. ox-LDL. (B) Proteins from HUVECs of ox-LDL-treated (ox-LDL) group, atorvastatin treated plus ox-LDL-treated (atorvastatin) group and AICAR treated plus ox-LDL-treated (AICAR) group were analyzed by western blot analysis. AMPK, sXBP-1 and p-eIF2a protein levels were examined and quantified. "P<0.05 compared with ox-LDL group. sXBP-1, sliced $\mathrm{x}$-box binding protein 1; eIF2a, eukaryotic initiation factor; PERK, protein kinase-like ER kinase; ERS, endoplasmic reticulum stress. 

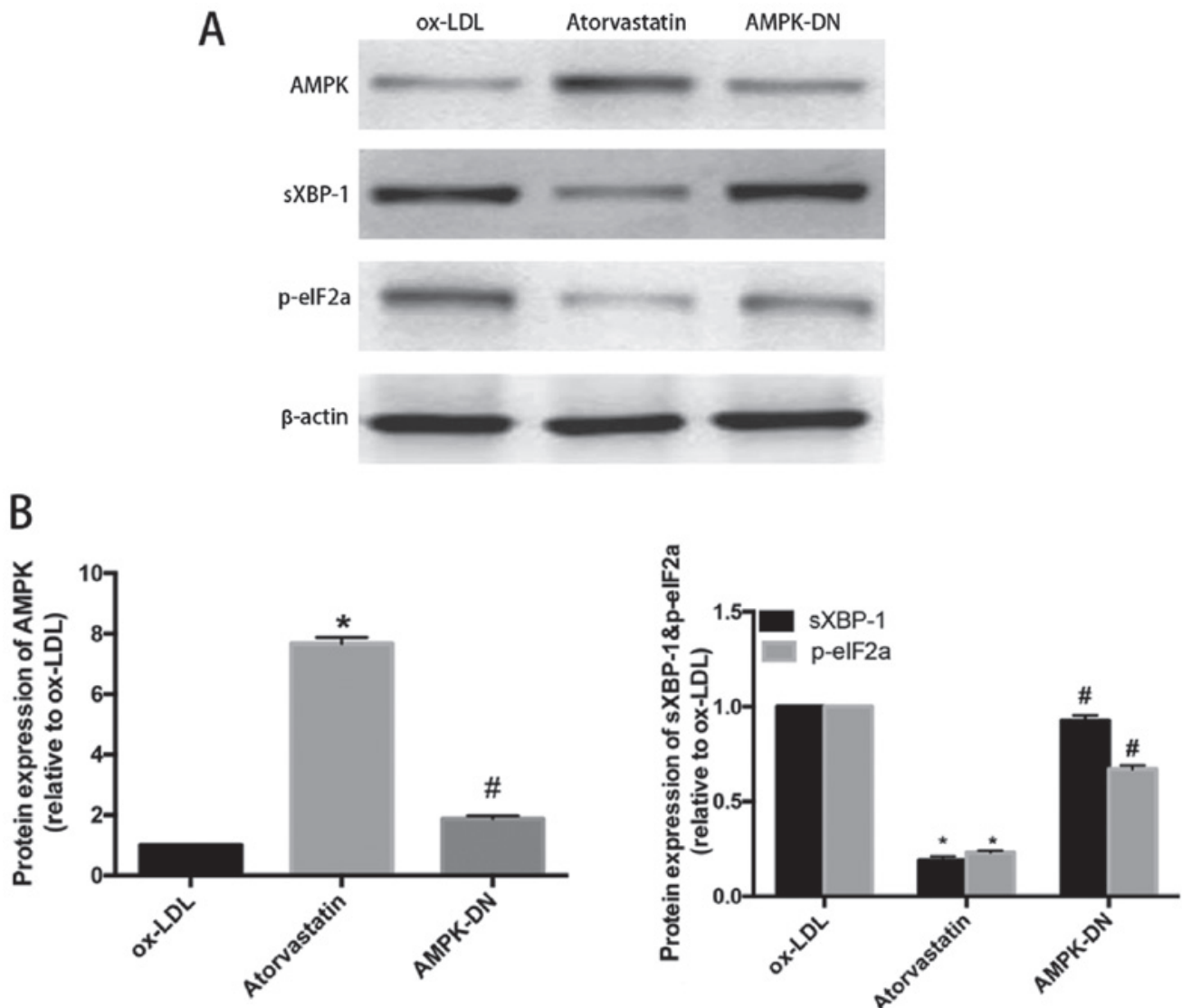

Figure 4. Atorvastatin attenuates oxidized modified low-density lipoprotein (ox-LDL)-induced ERS in human umbilical vein endothelial cells (HUVECs) through activating AMPK. (A) Proteins from HUVECs of ox-LDL-treated (ox-LDL) group, atorvastatin treated plus ox-LDL-treated (atorvastatin) group and AMPK-DN treated plus ox-LDL-treated (AMPK-DN) group were analyzed by western blot analysis. AMPK, sXBP-1 and p-eIF2a protein levels were examined and normalized with $\beta$-actin. (B) AMPK, sXBP-1 and p-eIF2a protein levels were quantified. "P<0.05 atorvastatin vs. ox-LDL; "P<0.05 AMPK-DN vs. atorvastatin. sXBP-1, sliced x-box binding protein 1; eIF2a, eukaryotic initiation factor; PERK, protein kinase-like ER kinase; ERS, endoplasmic reticulum stress.

initiation factor $2 \alpha(\mathrm{eIF} 2 \alpha)$, thereby reducing the overall level of protein translation and lowering the ER unfolded protein load (14). IRE1 is type I ER transmembrane protein with dual activity of serine/threonine kinase and endonuclease. After dissociation from GRP78, IRE1 forms dimers and leads to activation of its protein kinase activity and autophosphorylation, thereby activating its endonuclease activity. Then a 26 bp intron in XBP1 precursor mRNA is sliced, producing the active sXBP-1 (XBP1S). XBP1S enters the nucleus to bind to the promoter of ERS response element (ERSE), inducing the expression of molecular chaperones and foldase genes, and up-regulate the ER-associated degradation (ERAD) pathway-related proteins, thus promoting the correct folding and maturation of protein and the degradation of misfolded proteins (15).

Recent studies have shown that the ERS response exists throughout the occurrence and development of AS, participates in the activity regulation and apoptosis of vascular endothelial cells, vascular smooth muscle cells and macrophages, and plays an important role in AS caused by such risk factors such as hyperlipidemia, homocysteine and hyperglycemia (16-19). The activation of AMPK is associated with phosphorylation of eIF $2 \alpha$, and studies have revealed that atorvastatin can activate AMPK and reduce homocysteine-induced ERS response, thereby alleviating vascular wall damage and progression of AS (20).
In conclusion, this study confirmed that atorvastatin can relieve ERS and reduce atherosclerotic plaque formation in $\mathrm{ApoE}^{-/}$mice fed with high-fat diets. Atorvastatin exerts its inhibitory effect on ERS through AMPK, but its further signaling mechanism still needs in-depth study.

\section{Acknowledgements}

Not applicable.

\section{Funding}

No funding was received.

\section{Availability of data and materials}

All data generated or analyzed during this study are included in this published article.

\section{Authors' contributions}

WX, MF and ZZ designed the study and performed the experiments, WX and CW established the animal models, MF and WW collected the data, RL and LS analyzed the data, WX, MF and ZZ prepared the manuscript. All authors read and approved the final manuscript. 


\section{Ethics approval and consent to participate}

This study was approved by the Animal Ethics Committee of Fudan University Animal Center (Qingpu, China).

\section{Patient consent for publication}

Not applicable.

\section{Competing interests}

The authors declare they have no competing interests.

\section{References}

1. Zhang ZY,Hu CF, Wang MX, Lin J, Li JM and Wang RZ: Research on mechanism of PCS in damaging vascular endothelial cells and promoting formation of atherosclerosis via TLR4/TREM-1. Eur Rev Med Pharmacol Sci 22: 7533-7542, 2018.

2. Williams KJ and Tabas I: The response-to-retention hypothesis of atherogenesis reinforced. Curr Opin Lipidol 9: 471-474, 1998.

3. Tabas I: Macrophage death and defective inflammation resolution in atherosclerosis. Nat Rev Immunol 10: 36-46, 2010.

4. Moore KJ and Tabas I: Macrophages in the pathogenesis of atherosclerosis. Cell 145: 341-355, 2011

5. Kedi X, Ming Y, Yongping W, Yi Y and Xiaoxiang Z: Free cholesterol overloading induced smooth muscle cells death and activated both ER- and mitochondrial-dependent death pathway. Atherosclerosis 207: 123-130, 2009.

6. Nakano T, Watanabe H, Ozeki M, Asai M, Katoh H, Satoh H and Hayashi H: Endoplasmic reticulum $\mathrm{Ca}^{2+}$ depletion induces endothelial cell apoptosis independently of caspase-12. Cardiovasc Res 69: 908-915, 2006

7. Levine YC, Li GK and Michel T: Agonist-modulated regulation of AMP-activated protein kinase (AMPK) in endothelial cells. Evidence for an AMPK $\rightarrow$ Racl $->$ Akt $->$ endothelial nitric-oxide synthase pathway. J Biol Chem 282: 20351-20364, 2007.

8. Wang Y, Huang Y, Lam KS, Li Y, Wong WT, Ye H, Lau CW, Vanhoutte PM and Xu A: Berberine prevents hyperglycemiainduced endothelial injury and enhances vasodilatation via adenosine monophosphate-activated protein kinase and endothelial nitric oxide synthase. Cardiovasc Res 82: 484-492, 2009.

9. Bao XM, Wu CF and Lu GP: Atorvastatin inhibits homocysteineinduced oxidative stress and apoptosis in endothelial progenitor cells involving Nox4 and p38MAPK. Atherosclerosis 210 114-121, 2010.
10. Heeba G, Hassan MK, Khalifa M and Malinski T: Adverse balance of nitric oxide/peroxynitrite in the dysfunctional endothelium can be reversed by statins. J Cardiovasc Pharmacol 50: 391-398, 2007.

11. Arad M, Seidman CE and Seidman JG: AMP-activated protein kinase in the heart: Role during health and disease. Circ Res 100: 474-488, 2007.

12. Hetz C: The unfolded protein response: Controlling cell fate decisions under ER stress and beyond. Nat Rev Mol Cell Biol 13: 89-102, 2012.

13. Minamino T, Komuro I and Kitakaze M: Endoplasmic reticulum stress as a therapeutic target in cardiovascular disease. Circ Res 107: 1071-1082, 2010

14. Ma Y and Hendershot LM: Delineation of a negative feedback regulatory loop that controls protein translation during endoplasmic reticulum stress. J Biol Chem 278: 34864-34873, 2003.

15. Lerner AG, Upton JP, Praveen PV, Ghosh R, Nakagawa Y, Igbaria A, Shen S, Nguyen V, Backes BJ, Heiman M, et al: IRE1a induces thioredoxin-interacting protein to activate the NLRP3 inflammasome and promote programmed cell death under irremediable ER stress. Cell Metab 16: 250-264, 2012.

16. Ishiyama J, Taguchi R, Akasaka Y, Shibata S, Ito M, Nagasawa M and Murakami K: Unsaturated FAs prevent palmitate-induced LOX-1 induction via inhibition of ER stress in macrophages. J Lipid Res 52: 299-307, 2011.

17. Thorp E, Li G, Seimon TA, Kuriakose G, Ron D and Tabas I: Reduced apoptosis and plaque necrosis in advanced atherosclerotic lesions of $\mathrm{Apoe}^{-/-}$and $\mathrm{Ldlr}^{-/-}$mice lacking CHOP. Cell Metab 9: 474-481, 2009

18. Luo Y, Li SJ, Yang J, Qiu YZ and Chen FP: HMGB1 induces an inflammatory response in endothelial cells via the RAGE-dependent endoplasmic reticulum stress pathway. Biochem Biophys Res Commun 438: 732-738, 2013.

19. Larroque-Cardoso P, Swiader A, Ingueneau C, Nègre-Salvayre A, Elbaz M, Reyland ME, Salvayre R and Vindis C: Role of protein kinase C $\delta$ in ER stress and apoptosis induced by oxidized LDL in human vascular smooth muscle cells. Cell Death Dis 4: e520, 2013.

20. Jia F, Wu C, Chen Z and Lu G: Atorvastatin inhibits homocysteine-induced endoplasmic reticulum stress through activation of AMP-activated protein kinase. Cardiovase Ther 30: 317-325, 2012.

This work is licensed under a Creative Commons Attribution-NonCommercial-NoDerivatives 4.0 International (CC BY-NC-ND 4.0) License. 